\title{
Left atrial appendage closure in atrial fibrillation patients with prior major bleeding or ineligible for oral anticoagulation
}

\author{
L. I. S. Wintgens · V. M. M. Vorselaars · M. N Klaver · M. J. Swaans · A. Alipour · B. J. W. M. Rensing · M. C. Post • \\ L. V. A. Boersma
}

Published online: 11 June 2019

(c) The Author(s) 2019

\begin{abstract}
Aims Oral anticoagulation (OAC) reduces the ischaemic stroke risk in patients with atrial fibrillation (AF), but in turn leads to an increased risk of adverse bleeding events. Alternatively, left atrial appendage closure (LAAC) using a mechanical device might overcome these bleeding complications. However, evidence regarding LAAC in patients at high bleeding risk is scarce. This study evaluates the clinical features of AF patients with previous bleeding that underwent LAAC.

Methods In this retrospective cohort study patients with previous major bleeding or a bleeding predisposition scheduled for transcatheter LAAC were included. The frequency and type of previous bleeding events and prevalence of bleeding and ischaemic stroke during follow-up were evaluated.

Results A total of 73 patients $(58 \%$ male, age $72.1 \pm$ 7.2 years; $\mathrm{CHA}_{2} \mathrm{DS}_{2}$-VASc 4.5 [3.0-5.0]; HAS-BLED 4.0 [3.0-4.0]; 46\% paroxysmal AF) were included. Previous bleeding occurred from intracranial ( $n=50,69 \%)$, gastro-intestinal $(n=13,18 \%)$ or multiple $(n=16,22 \%)$ foci. After OAC discontinuation due to bleeding, $19 \%$ suffered subsequent stroke. LAAC was successful in $96 \%$ of patients. During a median of almost 3 years'
\end{abstract}

L. I. S. Wintgens $(\bowtie) \cdot$ V. M. M. Vorselaars $\cdot$ M. N. Klaver

M. J. Swaans · A. Alipour · B. J. W. M. Rensing · M. C. Post · L. V. A. Boersma

Department of Cardiology, St. Antonius Hospital,

Nieuwegein, The Netherlands

l.wintgens@antoniusziekenhuis.nl

\section{A. Alipour}

Department of Cardiology, Rivierenland Hospital, Tiel, The Netherlands

\section{V. A. Boersma}

Department of Cardiology, AMC Amsterdam, Amsterdam, The Netherlands follow-up recurrent major bleeding occurred in 4 patients $(5.5 \%)$ despite OAC discontinuation in $93.2 \%$. A total of 6 ischaemic strokes were observed, resulting in an annualised stroke rate of $2.9 \%$ compared to a calculated expected stroke rate of $6.7 \%$.

Conclusions Percutaneous LAAC may provide an alternative strategy to long-term OAC therapy in AF patients with a high bleeding risk. During follow-up, both ischaemic stroke and recurrent bleeding rates were lower than expected based on the $\mathrm{CHA}_{2} \mathrm{DS}_{2}$-VASc and HAS-BLED scores respectively.

Keywords Atrial fibrillation - Left atrial appendage closure · Catheter ablation - Stroke prevention · Bleeding

\section{What's new?}

- Left atrial appendage closure (LAAC) provides an alternative to long-term oral anticoagulation (OAC) for stroke prevention in atrial fibrillation (AF) patients.

- LAAC may reduce stroke risk and eliminate the need for OAC therapy in patients ineligible for OAC.

- We present one of the larger registries of patients with previous major bleeding or very high bleeding risk that underwent transcatheter LAAC.

- During long-term follow-up, stroke and bleeding rates were lower than expected based on $\mathrm{CHA}_{2} \mathrm{DS}_{2}$-VASc and HAS-BLED scores respectively, despite discontinuation of OAC in $93.2 \%$ of patients.

- Percutaneous LAAC may provide an alternative strategy to long-term OAC therapy in AF patients with a very high bleeding risk or a history of major bleeding. 


\section{Introduction}

Thromboembolic clots formed in the left atrial appendage account for up to $90 \%$ of atrial fibrillation (AF)-related strokes in patients with AF [1]. Although oral anticoagulation (OAC) therapy with vitamin $\mathrm{K}$ antagonists (VKA) significantly reduces the risk of stroke, this is associated with an increased risk of adverse (major) bleeding events. Even the non-VKA OACs (NOACs) are associated with an increased bleeding risk [2-6]. Moreover, patients with a history of major bleeding or contra-indications for OAC have always been excluded from the randomised trials.

Stroke prevention strategies are particularly challenging in patients in whom (N)OAC is contra-indicated. The need for OAC should be based on a careful individualised assessment of both stroke and bleeding risk, indicated as $\mathrm{CHA}_{2} \mathrm{DS}_{2}$-VASc and HAS-BLED score respectively [7]. Left atrial appendage closure (LAAC) using a mechanical device can provide an alternative to lifelong anticoagulation. Two large-scale randomised controlled trials have demonstrated the safety and efficacy of the Watchman device compared to long-term OAC therapy for stroke prevention $[8,9]$. However, in these trials only patients without contraindications for long-term OAC were included. Evidence for LAAC in patients with a history of major bleeding under (N)OAC is scarce, yet this is expected in the designated patient population in whom this therapy may be considered in current ESC guidelines [10]. In this observational cohort study, we report the clinical features of a real-world series of consecutive patients with previous major bleeding or very high bleeding risk that underwent transcatheter LAAC in our centre. The aim of the study was to show the procedural efficacy and safety and long-term outcome data in this selected population of AF patients that seeks an alternative to OAC because of severe bleeding problems in the past, and for which there is so far very limited evidence in the literature.

\section{Methods}

In this retrospective single-centre cohort study, consecutive patients scheduled for percutaneous LAAC, with non-valvular AF and contra-indications to OAC therapy, including previous major bleeding and very high tendency to fall, were included. Patients who underwent stand-alone LAAC as well as patients scheduled for a combined procedure with catheter ablation (CA) followed by LAAC were included.

\section{Data collection}

Data on percutaneous LAAC are collected prospectively by means of a web-based database. We recorded the rate and type of previous bleeding events, management of anticoagulation after bleeding, peri-pro- cedural characteristics and prevalence of major bleeding and ischaemic stroke during follow-up.

\section{Patient and procedural management}

All patients visited a cardiologist-electrophysiologist for evaluation of eligibility for percutaneous LAAC. Patients with an indication for LAAC were evaluated in multidisciplinary team meetings.

Transoesophageal echocardiography (TOE) was performed in all patients prior to the procedure to evaluate the LAA anatomy and to exclude intracardiac thrombus.

LAAC could be performed by either of the available percutaneous techniques used in our centre, Watchman (Boston Scientific, Natick, MA, USA) or Amplatzer Amulet (Abbott, Minneapolis, MN, USA). The choice of technique was left to the physician's discretion.

If patients were scheduled for the combined procedure, CA was performed prior to LAAC. All patients underwent post-procedural chest radiography to confirm the proper position of the device.

\section{Follow-up}

All patients were seen by their treating cardiologistelectrophysiologist in the outpatient clinic 3 and 12 months after the procedure. In addition, regular telephone interviews were performed.

Rhythm monitoring was performed through electrocardiograms (ECGs) and Holter recordings at 6 and 12 months of follow-up. Patients with symptoms suggestive of recurrent atrial arrhythmias were encouraged to obtain symptom-driven ECG recordings.

\section{Antithrombotic therapy}

The recommended post-implant regimen consisted of (1) clopidogrel and aspirin for 1-3 months (for Amplatzer devices) and for 6 months (for Watchman devices) post-implantation, (2) aspirin indefinitely.

However, the choice and duration of post-implant antithrombotic medication was not mandatory and was left to the physician.

TOE was repeated between 45 and 60 days postprocedure to evaluate device position, residual flow and thrombus formation.

\section{Outcome}

In this observational cohort study, we report on the incidence of ischaemic stroke during clinical followup. In the absence of a control group, the observed stroke rate was compared to the predicted stroke rate based on the $\mathrm{CHA}_{2} \mathrm{DS}_{2}$-VASc score [11].

Secondary outcome included procedural success, peri-procedural complications up to 30 days, management of antithrombotic medication, freedom from 
atrial tachyarrhythmias and major bleeding during follow-up.

Major bleeding was defined as bleeding type 3 or greater according to the BARC criteria [12].

\section{Results}

\section{Patient selection and characteristics}

Between 2010 and 2016, a total of 135 patients were scheduled for percutaneous LAAC in our institution,

Table 1 Baseline and peri-procedural characteristics

\begin{tabular}{|l|c|}
\hline Total no. of patients & 73 \\
\hline Age, years \pm SD & $72.1 \pm 7.2$ \\
\hline Male & $42(58 \%)$ \\
\hline Paroxysmal AF & $32(44 \%)$ \\
\hline Persistent or long-standing persistent AF & $41(56 \%)$ \\
\hline CHADS $_{2}$ & $3.0[2.0-4.0]$ \\
\hline CHA $_{2}$ DS 2 -VASC & $4.5[3.0-5.0]$ \\
\hline HAS-BLED & $4.0[3.0-4.0]$ \\
\hline History of stroke & $30(41 \%)$ \\
\hline History of bleeding & $70(96 \%)$ \\
\hline Intracranial bleeding & $50(69 \%)$ \\
\hline Gastro-intestinal bleeding & $13(18 \%)$ \\
\hline Pulmonary bleeding & $2(3 \%)$ \\
\hline Other bleeding site & $17(33 \%)$ \\
\hline Multiple bleeding sites & $16(22 \%)$ \\
\hline Indefinite OAC withdrawal after bleeding & $63(86 \%)$ \\
\hline Stroke after OAC withdrawal & $14(19 \%)$ \\
\hline Stand-alone LAAC procedure & $45(61.6)$ \\
\hline Combined CA and LAAC & $28(38.4)$ \\
\hline Device type & \\
\hline Watchman & $69(94.5)$ \\
\hline Amplatzer Amulet & $4(5.5)$ \\
\hline Successful LAAC & $70(95.9)$ \\
\hline Complete LAA closure & $67(95.7)$ \\
\hline Minimal residual flow & $3(4.3)$ \\
\hline Number of devices used & $1.0[1.0-1.0]$ \\
\hline Total procedure time, min & $92 \pm 34$ \\
\hline Total procedure time stand-alone, min & $78 \pm 31$ \\
\hline Fluoroscopy time, min & $11 \pm 5$ \\
\hline Major 30-day complications & $6(8.2)$ \\
\hline Pericardial effusion & $0(0.0)$ \\
\hline Intracoronary air embolus & $1(1.4)$ \\
\hline Device embolisation & $2(2.7)$ \\
\hline Stroke & $1(1.4)$ \\
\hline TIA & $2(2.7)$ \\
\hline Death & $0(0.0)$ \\
\hline Minor 30-day complications & $3(4.1)$ \\
\hline Groin haematoma & $3(4.1)$ \\
\hline AF atrial fibrillation, CA catheter ablation, LAA left atrial appendage, \\
\hline LAAC left atrial appendage closure, OAC oral anticoagulation, TIA transient \\
\hline ischaemic attack & \\
\hline
\end{tabular}

of whom 73 were included in this study. Patient characteristics at baseline are shown in Tab. 1 .

\section{Previous bleeding, stroke, and antithrombotic management}

Details of the prior bleeding events are shown in Tab. 1 . Seventy patients $(96 \%)$ had a history of previous major bleeding. Three patients $(4 \%)$ were considered ineligible for OAC due to severe thrombocytopenia or elevated falling risk due to narcolepsy and post-dystrophic muscular dystrophy. In the majority of patients (86\%) oral antithrombotic therapy had been discontinued or never started. Fourteen patients (19\%) suffered a stroke after OAC withdrawal.

\section{Procedural characteristics}

Procedural characteristics are shown in Tab. 1. LAAC was performed as a stand-alone procedure in 45 of 73 patients $(62 \%)$. In 28 patients LAAC was combined with CA.

Implantation of the LAAC device was unsuccessful in 3 patients: one device dislocation to the left ventricular outflow tract during the procedure and 2 patients with unsuitable anatomy. In 70 patients $(95.9 \%)$ an LAAC device could be implanted, leading to successful LAAC in all 70.

Serious peri-procedural complications up to 30 days occurred in $6(8.2 \%)$ patients (2 patients with a combined procedure, 4 patients with a stand-alone LAAC procedure).

Peri-procedural dislocation of the Watchman device occurred in 2 patients $(2.7 \%)$; in one patient a new device could be successfully implanted in the LAA. Two transient ischaemic attacks (TIAs) and one ischaemic stroke were reported to occur within $24 \mathrm{~h}$ of the procedure. TOE was performed in all these patients, showing complete closure of the LAA with no residual flow and no device thrombus. In the patient with a stroke, left atrial thrombus had been seen on TOE after the ablation sheath was exchanged for the Watchman sheath. Despite an extra 5,000 IU of heparin, this patient suffered an ischaemic stroke hours after the procedure with residual complaints. No periprocedural major bleeding, pericardial effusion, tamponade or death occurred. Minor groin haematoma was seen in 3 patients (4.1\%).

\section{Transoesophageal echocardiographic follow-up}

TOE was performed within 45-60 days in 69 of 70 (98.6\%) patients with a successfully implanted LAAC device, showing successful sealing of the LAA in 68 of $69(98.5 \%)$ and significant residual flow in 1 of 69 . No device thrombi were found. 
Table 2 Antithrombotic therapy management

\begin{tabular}{|l|c|c|c|}
\hline & Pre-procedural & 3 months & End of FU \\
\hline VKA & $8(11.0 \%)$ & $5(6.8 \%)$ & $2(2.7 \%)$ \\
\hline NOAC & $1(1.4 \%)$ & $1(1.4 \%)$ & $3(4.1 \%)$ \\
\hline SAPT & $0(0.0 \%)$ & $16(21.9 \%)$ & $6(8.2 \%)$ \\
\hline None & $36(49.3 \%)$ & $45(61.6 \%)$ & $52(71.2 \%)$ \\
\hline & $28(38.3 \%)$ & $6(8.2 \%)$ & $10(13.7 \%)$
\end{tabular}

VKA vitamin $\mathrm{K}$ antagonist, NOAC non-vitamin $\mathrm{K}$ antagonist oral anticoagulation, DAPT dual antiplatelet therapy, SAPT single antiplatelet therapy, FU follow-up

\section{Antithrombotic therapy management}

Table 2 shows pre- and post-implant oral antithrombotic therapy in all 73 patients.

After 35.5 months of follow-up, only 5 patients were still receiving VKA (2) or NOAC (3) therapy. Fifty-eight patients were on either single $(71 \%)$ or dual $(8 \%)$ antiplatelet therapy, while 10 patients (14\%) were receiving no antithrombotic medication at all.

\section{Clinical outcome}

Table 3 shows the clinical outcome of the patients after a median follow-up period of 35.5 months.

\section{Thromboembolic events}

One peri-procedural stroke $(1.4 \%)$ was observed within 30 days of LAAC implantation. From 30 days until 35.5 months of follow-up, a total of 5 ischaemic strokes $(6.8 \%)$ were recorded. The overall stroke rate was 6 of 73 included patients and 6 of 70 patients after successful LAAC implantation. This results in an annualised stroke rate of $2.9 \%$. The annualised stroke risk of $2.9 \%$ was compared to an estimated stroke rate of $6.7 \%$ based on $\mathrm{CHA}_{2} \mathrm{DS}_{2}$-VASc score [11], accounting for a $57 \%$ risk reduction.

In 3 of 5 patients TOE data after TIA or stroke were available, showing complete closure in 2 patients and

Table 3 Clinical outcome after 35.5 months of follow-up

\begin{tabular}{|l|l|}
\hline No. of patients & 73 \\
\hline Minor bleeding & $2(2.7)$ \\
\hline Epistaxis & $1(1.4)$ \\
\hline Subcutaneous haematoma & $1(1.4)$ \\
\hline Major bleeding & $4(5.5)$ \\
\hline Intracranial & $1(1.4)$ \\
\hline Gastro-intestinal & $2(2.7)$ \\
\hline Pulmonary & $1(1.4)$ \\
\hline Annualised bleeding rate & $1.8 \%$ \\
\hline Overall ischaemic stroke & $6(8.2)$ \\
\hline $\begin{array}{l}\text { Ischaemic stroke in patients with successfully implanted LAAC } \\
\text { device }\end{array}$ & $6(8.5)$ \\
\hline $\begin{array}{l}\text { Ischaemic stroke in patients with successfully implanted LAAC } \\
\text { device and cessation of (N)OAC }\end{array}$ & $6(9.0)$ \\
\hline Annualised stroke rate & 3.0 \\
\hline
\end{tabular}

minimal residual flow of $2 \mathrm{~mm}$ in 1 , and no device thrombus. Notably, 4 patients continued NOAC or OAC therapy owing to repeated electrical cardioversions and were thus at lower stroke risk. When these patients were excluded from the stroke risk analysis, the annualised stroke risk is $3.0 \%$.

All patients were on antiplatelet therapy at the time of stroke (5 on single antiplatelet therapy, 1 on dual antiplatelet therapy).

\section{Bleeding events}

Three patients (4.1\%) had four major bleeding events (5.5\%) from 30 days until 35.5 months of follow-up, resulting in an annualised bleeding event rate of $1.8 \%$. Bleeding occurred at the following foci: intracranial $(n=1)$, gastro-intestinal $(n=2)$ and pulmonary $(n=1)$. There was a relative risk reduction of $80 \%$ between the observed major bleeding rate of $1.8 \%$ and the expected bleeding rate of $8.9 \%$ under (N)OAC use. No fatal bleeding events occurred during follow-up.

\section{Atrial fibrillation recurrence}

Recurrent AF was seen in 19 of 28 patients that underwent combined CA and LAAC (67.9\%).

\section{Discussion}

This study reports the clinical features of a real-world series of patients with a very high bleeding risk that underwent transcatheter LAAC in our centre. In our population previous bleeding most often occurred (69\%) from intracranial foci, while after OAC discontinuation to avoid bleeding $19 \%$ of patients suffered from ischaemic stroke. LAAC was found to be feasible in this very vulnerable patient cohort, with stroke and bleeding rates during 3 years of follow-up much lower than expected based on $\mathrm{CHA}_{2} \mathrm{DS}_{2}$-VASc and HASBLED scores respectively.

The long-term benefit of stroke and bleeding reduction by LAAC therefore appears to outweigh the procedural risks and seems preferable to both avoidance and continuation of OAC.

Current guidelines state that LAAC may be considered in AF patients with contra-indications for long-term OAC therapy [10]. However, in the randomised PROTECT-AF and PREVAIL trials, which demonstrated the safety and efficacy of Watchman LAAC, patients with prior bleeding were not included $[8,9]$. Even in recent large real-world registries of LAAC less than half of the patients had an actual history of prior bleeding, and no sub-analysis has been performed for this population thus far [13, 14].

The only data focussing on this particular population come from smaller registries and are actually scarce [5]. Several small "real-world" cohort studies using various percutaneous devices [16-20] have indicated that LAAC appears to be feasible and safe in patients with a history of major bleeding of intracranial or gastro-intestinal origin. Our present study with 


\title{
Advertisement placed here.
}

\author{
ces bohn \\ CL van loghum
}

Houten 2019 


\title{
Advertisement placed here.
}

\author{
ces bohn \\ CL van loghum
}

Houten 2019 
$96 \%$ of patients with a history of major bleeding is in line with those data and represents a larger cohort of patients that were not selected according to type of bleeding.

\section{The therapeutic dilemma in contra-indicated patients}

The HAS-BLED score is widely used to estimate the risk of major bleeding in patients with AF. However, it entails important drawbacks, including limited sensitivity and specificity and the fact that it does not account for type of bleeding or recurrence rate. Patients who have suffered from multiple bleeding foci and/or multiple bleeding events, as $22 \%$ of patients in our cohort did, clearly have a much higher risk of future recurrent bleeding, which is not reflected as an increase in their HAS-BLED score.

Furthermore, as CHADS-VASc and HAS-BLED scores share several risk factors, individual bleeding and stroke risk increase in parallel, creating a therapeutic dilemma in patients with very high risk of both stroke and bleeding.

Such patients are often forced to discontinue all antithrombotic medication, leaving them with a substantially increased stroke risk. Indeed, our study found that $19 \%$ of patients suffered from ischaemic stroke after OAC was withdrawn owing to previous bleeding. A strategy of LAAC with an annualised stroke rate of only $2.9 \%$ therefore appears to be more effective than providing these patients with no alternative to anticoagulation.

Previous studies have reported major bleeding in the peri-procedural period ranging from $1.0 \%$ to $5.1 \%$ $[8,13,21]$.

In our cohort of patients with a history of major bleeding, we did not observe a single peri-procedural major bleeding event. Only minor groin haematomas that could be managed conservatively were observed in $4.1 \%$. The operators were experienced in cardiac catheterisation and left atrial procedures, and these contra-indicated patients in general had lower periprocedural anticoagulation use. However, an overall complication rate of $8.2 \%$ was found, which is in line with the PROTECT-AF and ASAP registries but slightly higher than in the PREVAIL and EWOLUTION registries $[8,13,15,21]$. The less aggressive antithrombotic strategies in the peri-procedural phase may have contributed to the peri-procedural thromboembolism rate.

In our series the actual annualised stroke risk of $2.9 \%$ compared favourably to an estimated stroke risk of $6.7 \%$ based on $\mathrm{CHA}_{2} \mathrm{DS}_{2}$-VASc score, constituting a $57 \%$ risk reduction.

This is in line with the $62 \%$ ischaemic stroke risk reduction observed in the PREVAIL registry, and only slightly lower than the ischaemic stroke risk reductions in the PROTECT-AF, CAP and EWOLUTION registries $[8,22,23]$.

\section{Limitations}

This study is subject to the limitations associated with a retrospective study design, including the lack of a control group and a relatively small sample size.

We emphasise that our study was inevitably subject to selection bias, including only patients that were referred for LAAC. AF-related ischaemic strokes as well as OAC-related bleeding events have been associated with high morbidity and mortality [24]. Naturally, this patient cohort consisted only of patients who survived a major bleeding event and were eligible to undergo LAAC. Patients who subsequently died or suffered from severe (neurological) deficit were probably not referred to our centre. Therefore, there is a probability that the magnitude of this problem could have been larger than reported in our study.

Furthermore, the study cohort consisted of a heterogeneous patient group at vastly differing risks for (recurrent) bleeding and stroke. Consequently, different LAAC devices were used and various pre- and post-implant antithrombotic strategies were applied.

Future larger prospective and randomised controlled trials are needed to provide more knowledge on LAAC in this challenging subgroup of patients. They are among patients with the highest stroke and bleeding risk and are therefore expected to benefit most from this procedure.

\section{Conclusion}

Percutaneous LAAC may provide an alternative strategy to long-term OAC therapy in AF patients with a very high bleeding risk due to previous major bleeding. During follow-up, both ischaemic stroke and recurrent bleeding rates were lower than expected based on the $\mathrm{CHA}_{2} \mathrm{DS}_{2}$-VASc and HAS-BLED scores respectively.

Funding Left atrial appendage closure is not reimbursed in the Netherlands. Therefore, the procedures were financed by a research funding grant from the St. Antonius Hospital, allowing up to a maximum of 30 procedures per year. L. Wintgens received funding from ZonMW (ZonMW Topzorg).

Conflict of interest L.V.A. Boersma is a consultant for Medtronic, Abbott and Boston Scientific; fees go to the Cardiology Department. The Cardiology Department of the St. Antonius Hospital in Nieuwegein, the Netherlands receives proctoring fees for training/educational services from Boston Scientific. L.I.S. Wintgens, V.M.M. Vorselaars, M.N. Klaver, M.J. Swaans, A. Alipour, B.J.W.M. Rensing and M.C. Post declare that they have no competing interests.

Open Access This article is distributed under the terms of the Creative Commons Attribution 4.0 International License (http://creativecommons.org/licenses/by/4.0/), which permits unrestricted use, distribution, and reproduction in any medium, provided you give appropriate credit to the original author(s) and the source, provide a link to the Creative Commons license, and indicate if changes were made. 


\section{References}

1. Blackshear JL, Odell JA. Appendage obliteration to reduce stroke in cardiac surgical patients with atrial fibrillation. Ann ThoracSurg. 1996;61:755-9. Feb.

2. Ruff CT, Giugliano RP, Braunwald E, et al. Comparison of the efficacy and safety of new oral anticoagulants with warfarin in patients with atrial fibrillation: a meta-analysis of randomised trials. Lancet. 2014;383:955-62.

3. Patel MR, Mahaffey KW, Garg J, Pan G, et al. Rivaroxaban versus warfarin in nonvalvular atrial fibrillation. N Engl J Med. 2011;365:883-91.

4. Connolly SJ, Ezekowitz MD, Yusuf S, et al. Dabigatran versus warfarin in patients with atrial fibrillation. N Engl J Med. 2009;361:1139-51.

5. Granger CB, Alexander JH, McMurray JJ, et al. Apixaban versus warfarin in patients with atrial fibrillation. N Engl J Med. 2011;365:981-92.

6. Giugliano RP, Ruff CT, Braunwald E, et al. Edoxaban versus warfarin in patients with atrial fibrillation. N Engl J Med. 2013;369:2093-104.

7. Lip GYH, Andreotti F, Fauchier L, et al. Bleeding risk assessment and management in atrial fibrillation patients: a position document from the European Heart Rhythm Association, endorsed by the European Society of Cardiology Working Group on Thrombosis. Europace. 2011;13:723-46.

8. ReddyVY, SievertH, Halperin J, etal. Percutaneousleft atrial appendage closure vs warfarin for atrial fibrillation. JAMA. 2014;312:1988-98.

9. Holmes DR, Kar S, Price MJ, et al. Prospective randomized evaluation of the Watchman Left Atrial Appendage Closure device in patients with atrial fibrillation versus long-term warfarin therapy: the PREVAIL trial. J Am Coll Cardiol. 2014;64:1-12.

10. Kirchhof P, Benussi S, Kotecha D, et al. ESC Guidelines for the management of atrial fibrillation developed in collaboration with EACTS. Europace. 2016;2016(18):1609-78.

11. Friberg L, Rosenqvist M, Lip GYH. Evaluation of risk stratification schemes for ischaemic stroke and bleeding in 182678 patients with atrial fibrillation: the Swedish Atrial Fibrillation cohort study. Eur HeartJ. 2012;33:1500-10.

12. Mehran R, Rao SV, Bhatt DL, et al. Standardized bleeding definitions for cardiovascular clinical trials: a consensus report from the bleeding academic research consortium. Circulation. 2011;123(23):2736-47.

13. BoersmaLVA, SchmidtB, BettsTR, etal. Implantsuccessand safety of left atrial appendage closure with the WATCHMAN device: peri-procedural outcomes from the EWOLUTION registry. Eur Heart J. 2016;37:2465-74.
14. Tzikas A, Shakir S, Gafoor S, et al. Left atrial appendage occlusion for stroke prevention in atrial fibrillation: multicentre experience with the AMPLATZER Cardiac Plug. EuroIntervention. 2016;11:1170-9.

15. Reddy VY, Möbius-Winkler S, Miller MA, et al. Left atrial appendage closure with the Watchman device in patients with a contraindication for oral anticoagulation: the ASAP study (ASA Plavix Feasibility Study With Watchman Left Atrial Appendage Closure Technology). J Am Coll Cardiol. 2013;61:2551-6.

16. Kebernik J, Jose J, Abdel-Wahab M, Stöcker B, Geist V, Richardt G. Safety and efficacy of left atrial appendage closure with the Amplatzer cardiac plug in very high stroke and bleeding risk patients with non-valvular atrial fibrillation. Cardiol Ther. IEEE Trans Med Imaging. 2015;4:167:77.

17. Lempereur M, Aminian A, Freixa X, et al. Left atrial appendage occlusion in patients with atrial fibrillation and previous major gastrointestinal bleeding (from the Amplatzer Cardiac Plug Multicenter Registry). AmJ Cardiol. 2017;120:414-20.

18. Fahmy P, Spencer R, Tsang M, Gooderham P, Saw J. Left atrial appendage closure for atrial fibrillation is safe and effective after intracranial or intraocular hemorrhage. Can J Cardiol. 2016;32:349-54.

19. Horstmann S, Zugck C, Krumsdorf U, et al. Left atrial appendage occlusion in atrial fibrillation after intracranial hemorrhage. Neurology. 2014;82:135-8.

20. Tzikas A, Freixa X, Llull L, et al. Patients with intracranial bleeding and atrial fibrillation treated with left atrial appendage occlusion: results from the Amplatzer Cardiac Plug registry. Int J Cardiol. 2017;236:232-6.

21. Holmes DR, Reddy VY, Turi ZG, et al. Percutaneous closure of the left atrial appendage versus warfarin therapy for prevention of stroke in patients with atrialfibrillation: a randomised non-inferiority trial. Lancet. 2009;374:534-42.

22. Gangireddy SR, Halperin JL, Fuster V, Reddy VY. Percutaneous left atrial appendage closure for stroke prevention in patients with atrial fibrillation: an assessment of net clinical benefit. Eur HeartJ. 2012;33:2700-8.

23. Boersma LV, Ince H, Kische S, et al. Efficacy and safety of left atrial appendage closure with WATCHMAN in patients with or without contraindication to oral anticoagulation: 1-year follow-up outcome data of the EWOLUTION trial. Heart Rhythm. 2017;14:1302-8.

24. Fang MC, Go AS, Chang Y, et al. Death and disability from warfarin-associated intracranial and extra-cranial hemorrhages. Am J Med. 2007;120(8):700-5. 